\title{
Hepatitis Autoinmune en niños en un Hospital de Tercer Nivel. Reporte de casos
}

\section{Autoimmune Hepatitis in Children in a Tertiary Care Hospital: Report of a Series of Cases}

\author{
Rocío Morínigo $^{(1)}$, Jorge Ortiz ${ }^{(1)}$, Zoilo Morel Ayala ${ }^{(2)}$, Lidia Garcete ${ }^{(3)}$, Lourdes Ortiz Paranza ${ }^{(3)}$, Roger \\ Rolón $^{(1)}$
}

\begin{abstract}
RESUMEN
Introducción: La Hepatitis autoinmune (HAI) en niños se constituye en una enfermedad inflamatoria del hígado, caracterizada histológicamente por un denso infiltrado de células mononucleares en el sistema porta, y serológicamente por la presencia de auto-anticuerpos específicos del hígado y aumento de los niveles de transaminasas e IgG, en ausencia de una patología conocida que pueda afectar el hígado. Casos Clínicos: Se presenta un total de 10 casos con diagnóstico de HAI, la media de edad es de 12 años, con 2 de sexo femenino (20\%) y 8 masculino (80\%); 4 casos fueron de tipo 1 , un caso de tipo 2, y a 5 pacientes no se los pudo clasificar. La elevación de transaminasas ( $\geq$ x10 su valor normal) se observó en el $70 \%$ de los casos y la hipergammaglobulinemia en todos los casos. El tratamiento instaurado consistió en corticosteroides y azatioprina en todos los pacientes, con un tiempo medio de remisión de 4-6 semanas. Se observó abandono de tratamiento en 3 casos de los 10 casos, 2 de ellos con desenlace fatal. Discusión: La HAI presenta manifestaciones clínicas generales y específicas en etapas tempranas de la enfermedad. El alto porcentaje de respuesta al tratamiento médico nos hace considerar que el diagnóstico y tratamiento precoz son muy importantes, con el objetivo de detener la evolución a la cirrosis.
\end{abstract}

Palabras clave: Hepatitis autoinmune, enfermedad hepática crónica, hipergammaglobulinemia.

\begin{abstract}
Introduction: Autoimmune hepatitis $(\mathrm{AIH})$ in children is an inflammatory disease of the liver, characterizad histologically by mononuclear cell infiltrates in the portal system, and serologically by the presence of liver-specific autoantibodies and increased transaminase and IgG levels in the absence of a known pathology that could affect the liver. Case Reports: We present 10 cases of patients with a mean age of 12 years diagnosed with $\mathrm{AIH}$, including 2 female (20\%) and 8 male ( $80 \%$ ) patients; 4 cases were of Type 1, 1 was Type 2, and 5 were unclassifiable. Elevated transaminase ( $\geq$ x10 normal value) was observed in $70 \%$, while hypergammaglobulinemia was seen in all cases. Treatment instituted was corticosteroids and azathioprine in all patients, with a mean time to remission of 4-6 weeks. Treatment elopement occurred in 3 cases, 2 of which resulted in death. Discussion: AIH presents general and specific clinical manifestations in early stages of the disease. The high response rate to medical treatment in our study leads us to suggest that early diagnosis and treatment are very important in preventing progression to cirrhosis.
\end{abstract}

Keywords: Autoimmune hepatitis, chronic liver disease, hypergammaglobulinemia.

\footnotetext{
1. Cátedra y Servicio de Pediatría, Hospital de Clínicas, Facultad de Ciencias Médicas, Universidad Nacional de Asunción. San Lorenzo, Paraguay.

2. Reumatología Pediátrica, Cátedra y Servicio de Pediatría, Hospital de Clínicas, Facultad de Ciencias Médicas, Universidad Nacional de Asunción. San Lorenzo, Paraguay.

3. Gastroenterología Pediátrica, Cátedra y Servicio de Pediatría, Hospital de Clínicas, Facultad de Ciencias Médicas, Universidad Nacional de Asunción. San Lorenzo, Paraguay.

Correspondencia: Dra. Rocío Morínigo. Hospital de Clínicas. Mcal. López c/ Cruzada de la Amistad. San Lorenzo, Paraguay. E-mail: ronchi_125@hotmail.com

Recibido: 25/01/2015; Aprobado: 20/03/2015.

http://dx.doi.org/10.18004/ped.2015.abril.48-53

Los autores declaran que no existen conflictos de interés en el presente estudio.
} 
La hepatitis autoinmune (HAI) de la infancia se constituye en una enfermedad inflamatoria del hígado, caracterizada histológicamente por un denso infiltrado de células mononucleares en el sistema porta, y serológicamente por la presencia de auto-anticuerpos específicos del hígado y aumento de los niveles de las transaminasas y la IgG, en ausencia de una patología conocida que pueda afectar el hígado. En los niños, a menudo se presenta en forma aguda y tiene un curso más agresivo que en los pacientes mayores. El requisito antiguo de una duración de síntomas de 6 meses antes del diagnóstico ha sido abandonado ${ }^{(1)}$.

Presenta una incidencia de 1-2 por 100.000 habitantes por año, una prevalencia de 10-20/100.000 habitantes y se observa en todos los grupos étnicos, es predominante en el sexo femenino en una proporción de 3,6:1. La mayor incidencia ocurre durante la adolescencia y entre los 35-40 años de edad ${ }^{(2,3)}$.

Se desconoce la causa principal de la HAI. Como en las demás patologías autoinmunes, la patogenia es el resultado entre la interacción de alteraciones en la tolerancia inmunológica, predisposición genética factores ambientales como disparadores. Se han descrito potenciales "triggers" a agentes infecciosos (virus del sarampión, virus de la hepatitis, citomegalovirus, y Virus de Epstein-Barr) y medicamentos (productos a base de hierbas, antibióticos, estatinas, anti-FNT) ${ }^{(4-6)}$.

El diagnóstico de HAI se basa en un sistema de puntuación desarrollado por el Grupo Internacional de Hepatitis Autoinmune en 1999 y revisado en $2010^{(7-9)}$ (Tabla 1).

La HAI puede presentarse en forma aguda, crónica, con hepatitis fulminante o con insuficiencia hepática progresiva.

En los estudios de laboratorio, es más frecuente encontrar elevación de transaminasas e hipergammaglobulinemia ${ }^{(10-12)}$.

La biopsia hepática ha sido durante años el método de referencia para cuantificar la fibrosis, no obstante es una técnica invasiva y con limitaciones importantes. Los hallazgos histológicos característicos corresponden a la hepatitis de interfase, con presencia de necrosis periportal con infiltrado linfoplasmocitario, aunque esto no se presenta siempre $^{(13,14)}$.

El tratamiento se basa en la inmunosupresión, a fin de evitar la progresión a cirrosis y falla hepática, ya que rara vez se presenta la remisión espontánea ${ }^{(12)}$.

El trasplante de hígado es un tratamiento efectivo para pacientes en etapas finales de enfermedad hepática incluida la hepatitis autoinmune. La recurrencia y la reaparición "de novo" de HAI en pacientes trasplantados ha sido reportado ${ }^{(15)}$.

En este trabajo presentamos la caracterización de la HAI en niños en la Cátedra y Servicio de Pediatría, Facultad de Ciencias Médicas (FCM), Universidad Nacional de Asunción (UNA).

\section{CASOS CLÍNICOS}

En esta serie de casos fueron incluidos pacientes con diagnóstico de hepatitis autoinmune, en la Cátedra y Servicio de Pediatría, FCM, UNA, en el periodo comprendido entre enero de 2006 y diciembre de 2013. Fueron excluidos los pacientes que presentaron otras causas que expliquen la hepatitis (causas metabólicas, genéticas o infecciosas), así como aquellos que fueron atendidos fuera del periodo estipulado. Se utilizaron historias clínicas del archivo del Servicio mencionado, previa solicitud a las autoridades correspondientes.

La frecuencia se determinó a través del número de pacientes que consultaron en dicho centro con el diagnóstico de hepatitis autoinmune durante el período de estudio. Los datos se recogieron a través de un cuestionario pre codificado con datos personales (edad, sexo), características clínicas de la enfermedad (peso, talla, ictericia, hepatomegalia, esplenomegalia, ascitis, encefalopatía, sangrados, coluria, acolia, fiebre, astenia, palidez, dolor abdominal), datos laboratoriales (hemoglobina, bilirrubina, transaminasas, tiempo de protrombina, eritrosedimentación, Test de Coombs directo, ANA, Anti-músculo liso, anti-LMK 1, serología para hepatitis virales), datos ecográficos y mejoría clínica y laboratorial luego de iniciado el tratamiento.

El estudio se llevó a cabo en un periodo de 8 años, entre enero de 2006 y diciembre de 2013. En dicho periodo, se registraron 17 pacientes con el 
diagnóstico de hepatopatía de etiología a determinar, de los cuales 59\% (10 casos) fueron hepatitis autoinmune, 29\% (5 casos) eran de etiología metabólica o genética, 11\% (2 casos) secundarios a infección por Citomegalovirus perinatal.

Fueron incluidos 10 pacientes con el diagnóstico de Hepatitis Autoinmune, en base al sistema de puntuación desarrollado por el Grupo Internacional de Hepatitis Autoinmune en 1999 y revisado en 2010, de los cuales 2 tuvieron presentación crónica y 8 en forma aguda, con falla hepática.

Se observó un predominio del sexo masculino con 8 varones $(80 \%)$ y 2 niñas (20\%). El rango de edad fue de 9 a 15 años, con una media de 12 años. A continuación se mencionan las características clínicas más relevantes al momento del diagnóstico, y los hallazgos en ecografía abdominal (Tablas 2 y 3).

Tabla 1. Sistema de puntuación simplificado del Grupo Internacional de Hepatitis Autoinmune ${ }^{(7-9)}$.

\begin{tabular}{llc}
\hline Anticuerpos & & Puntajes \\
\hline ANA o Anti-músculo liso & $\geq 40$ & +1 \\
ANA o Anti-músculo liso & $\geq 80$ & +2 \\
Anti-LKM 1 & $>40$ & +2 \\
Anti antigen hepático soluble & Positivo & +2 \\
Autoanticuerpos ausentes & Ninguno & 0 \\
Nivel de inmunoglobulina & & +1 \\
IgG & $>$ mayor al valor normal & +2 \\
& $>1.1$ mayor al valor normal & 0 \\
Histología & Normal & \\
Datos morfológicos de HAI & Compatible & +1 \\
& Típico & +2 \\
Enfermedad viral & Incompatible & 0 \\
Ausencia de hepatitis viral & Sin marcadores virales & +2 \\
& Marcadores virales + & 0 \\
Puntuación pre-tratamiento & Diagnóstico definitivo & $\mathbf{7}$ \\
& Diagnóstico probable & $\mathbf{6}$ \\
\hline
\end{tabular}

Tabla 2. Características clínicas de los pacientes al momento del diagnóstico. N: 10.

\begin{tabular}{|c|c|c|c|c|c|c|c|c|c|c|}
\hline & $\begin{array}{c}\text { Caso } \\
1 \\
\end{array}$ & $\begin{array}{c}\text { Caso } \\
2 \\
\end{array}$ & $\begin{array}{c}\text { Caso } \\
3 \\
\end{array}$ & $\begin{array}{c}\text { Caso } \\
4 \\
\end{array}$ & $\begin{array}{c}\text { Caso } \\
5 \\
\end{array}$ & $\begin{array}{c}\text { Caso } \\
6 \\
\end{array}$ & $\begin{array}{c}\text { Caso } \\
7\end{array}$ & $\begin{array}{c}\text { Caso } \\
\mathbf{8} \\
\end{array}$ & $\begin{array}{c}\text { Caso } \\
9 \\
\end{array}$ & $\begin{array}{c}\text { Caso } \\
10 \\
\end{array}$ \\
\hline EDAD (años) & 11 & 14 & 13 & 9 & 15 & 12 & 10 & 12 & 15 & 11 \\
\hline SEXO & M & M & M & $\mathrm{F}$ & M & $\mathrm{F}$ & M & M & M & M \\
\hline \multicolumn{11}{|c|}{ CLÍNICA } \\
\hline Ictericia & SI & & $\mathrm{SI}$ & SI & SI & $\mathrm{SI}$ & $\mathrm{SI}$ & $\mathrm{SI}$ & $\mathrm{SI}$ & $\mathrm{SI}$ \\
\hline Coluria & $\mathrm{SI}$ & & & $\mathrm{SI}$ & & & & $\mathrm{SI}$ & & \\
\hline Acolia & SI & & & $\mathrm{SI}$ & & & & & & \\
\hline Dolor Abdominal & & & SI & & & $\mathrm{SI}$ & & & & \\
\hline Vísceromegalia & & $\mathrm{SI}$ & & $\mathrm{SI}$ & & & & & $\mathrm{SI}$ & $\mathrm{SI}$ \\
\hline Hemorragia digest & stiva & SI & & & & & & & & \\
\hline Ascitis & & & $\mathrm{SI}$ & & & & & $\mathrm{SI}$ & & \\
\hline Encefalopatía & SI & & $\mathrm{SI}$ & & & & & & & \\
\hline Erupción cutánea & & & $\mathrm{SI}$ & & & & & & & \\
\hline Sangrados $(*)$ & $\mathrm{SI}$ & & & & & $\mathrm{SI}$ & $\mathrm{SI}$ & & & \\
\hline $\begin{array}{l}\text { ESTADO } \\
\text { NUTRICIONAL } \\
(+)\end{array}$ & $\begin{array}{l}\text { DCP } \\
\text { SEV }\end{array}$ & EUT & $\begin{array}{l}\text { DCP } \\
\text { SEV } \\
\end{array}$ & EUT & EUT & EUT & EUT & EUT & $\begin{array}{l}\text { DCP } \\
\text { SEV }\end{array}$ & EUT \\
\hline
\end{tabular}

Tabla 3: Hallazgos en ecografía abdominal al momento del diagnóstico. $\mathrm{N}: 10$.

\begin{tabular}{|c|c|c|c|c|c|c|c|c|c|c|}
\hline & $\begin{array}{c}\text { Caso } \\
1\end{array}$ & $\begin{array}{c}\text { Caso } \\
2\end{array}$ & $\begin{array}{c}\text { Caso } \\
3\end{array}$ & $\begin{array}{c}\text { Caso } \\
4\end{array}$ & $\begin{array}{c}\text { Caso } \\
5\end{array}$ & $\begin{array}{c}\text { Caso } \\
6\end{array}$ & $\begin{array}{c}\text { Caso } \\
7\end{array}$ & $\begin{array}{c}\text { Caso } \\
8\end{array}$ & $\begin{array}{c}\text { Caso } \\
9\end{array}$ & $\begin{array}{c}\text { Caso } \\
10\end{array}$ \\
\hline \multicolumn{11}{|c|}{ ECOGRAFÍA } \\
\hline Cirrocis $\left({ }^{\wedge}\right)$ & $\mathrm{SI}$ & SI & $\mathrm{SI}$ & NO & $\mathrm{SI}$ & NO & NO & $\mathrm{SI}$ & NO & $\mathrm{SI}$ \\
\hline Hipertensión Portal & $\mathrm{Al}$ & $\mathrm{SI}$ & $\mathrm{SI}$ & $\mathrm{NO}$ & $\mathrm{SI}$ & NO & SI & $\mathrm{SI}$ & NO & SI \\
\hline
\end{tabular}

En cuanto a las serologías para los virus de la hepatitis, 2 pacientes (20\%) presentaron antecedentes de infección previa por el virus de la Hepatitis A con serología positiva. En todos los pacientes se descartaron otras causas virales y Enfermedad de Wilson.

Los resultados de laboratorio, como aminotransferasas, bilirrubinas, TP, IgG y anticuerpos se expresan en la Tabla 4. Seguido de la tabla 5 donde se observan los parámetros de laboratorio luego del inicio del tratamiento, entre la $2^{\circ}$ y $3^{\circ}$ semana (primera columna) y posteriormente entre la $4^{\circ}$ y $6^{\circ}$ semana (segunda columna).

Tabla 4. Características de laboratorios de los pacientes. $\mathrm{N}: 10$.

\begin{tabular}{ccccccccc}
\hline Paciente & $\begin{array}{l}\text { GOT, GPT } \\
\text { x valor } \\
\text { normal (UI/L) }\end{array}$ & TP (\%) & $\begin{array}{c}\text { Bilirrubina } \\
(\mathbf{m g} / \mathbf{d l})\end{array}$ & IgG (\%) & ANA & ASMA & $\begin{array}{c}\text { Anti- } \\
\text { LKM } \mathbf{1}\end{array}$ & $\begin{array}{c}\text { HAI } \\
\text { tipo }\end{array}$ \\
\hline $\mathbf{1}$ & $\times 15$ & 26 & 3,38 & 59 & + & + & - & 1 \\
$\mathbf{2}$ & $\times 4$ & 61 & 1,1 & 27,6 & + & - & - & 1 \\
$\mathbf{3}$ & $\times 15$ & 28 & 4,2 & SD & - & - & SD & NO CLAS \\
$\mathbf{4}$ & $\times 50$ & 60 & 3 & 53 & - & + & - & 1 \\
$\mathbf{5}$ & $\times 2$ & 39 & 1,8 & 36 & - & - & - & NO CLAS \\
$\mathbf{6}$ & $\times 10$ & 58 & 1,2 & 38,8 & - & - & - & NO CLAS \\
$\mathbf{7}$ & $\times 5$ & 38 & 2,4 & SD & + & + & - & 1 \\
$\mathbf{8}$ & $\times 10$ & 70 & 1,19 & 39,7 & + & - & + & 2 \\
$\mathbf{9}$ & $\times 10$ & 64 & 4,49 & 30,9 & - & - & - & NO CLAS \\
$\mathbf{1 0}$ & $\times 15$ & 42 & 4,23 & 44,1 & - & - & - & NO CLAS \\
\hline
\end{tabular}

TP: tiempo de protrombina, ANA: anticueropos antinucleares, ASMA: anticuerpo anti musculo liso, LKM1 anticuerpos anti microsoma de hígado-riñón.

SD: sin datos, +: positivo, -: negativo)
Valores de referencia: BT: 0 - $1,00 \mathrm{mg} / \mathrm{dL}$. GOT: 0 - $32 \mathrm{UI/L}, \mathrm{GPT}: 0$ - $37 \mathrm{UI} / \mathrm{L}$. Tiempo de protombina: mayor a $60 \%$, Gamma globulina: 8 a $20,5 \%$.

En un paciente (10\%) (CASO 8) cuya clasificación inicial fue la de HAI tipo 2 se realizó el diagnóstico de Lupus Eritematoso Sistémico con nefritis en el periodo de seguimiento, con marcadores serológicos de autoinmunidad al momento del diagnóstico positivos para Test de Coombs Directo, ANA, ASMA y LMK-1, además de valores de C3 y C4 consumidos, artritis y anemia hemolítica autoinmune.

Ningún paciente presentó antecedentes familiares de enfermedades autoinmunes. En un solo paciente $(10 \%)$ se realizó biopsia hepática (CASO 2), con 
datos de cirrosis macronodular en la anatomía patológica. El $100 \%$ de los pacientes inició tratamiento con prednisona más azatioprina al momento del diagnóstico. La tabla 6 expresa la evolución de los pacientes.

Tabla 5. Resultados de laboratorio post-inicio del tratamiento, entre la $2^{\mathrm{o}}$ y $3^{\mathrm{o}}$ semana (primera columna), y entre la $4^{\circ}$ y $6^{\circ}$ semana (segunda columna).

\begin{tabular}{|c|c|c|c|c|c|c|c|c|c|c|c|c|c|c|c|c|c|c|c|}
\hline \multirow[b]{2}{*}{ BILIRRUBINA TOTAL } & \multicolumn{2}{|c|}{ Caso $1^{(1)}$} & \multicolumn{2}{|c|}{ Caso 2} & \multicolumn{2}{|c|}{ Caso $3^{(2)}$} & \multicolumn{2}{|c|}{ Caso 4} & \multicolumn{2}{|c|}{ Caso 5} & \multicolumn{2}{|c|}{ Caso 6} & \multicolumn{2}{|c|}{ Caso 7} & \multicolumn{2}{|c|}{ Caso 8} & \multicolumn{2}{|c|}{ Caso 9} & Caso 10 \\
\hline & 1,2 & $\mathrm{~S} / \mathrm{D}$ & 0,65 & 0,6 & 3,5 & $x$ & $\mathrm{~S} / \mathrm{D}$ & $\mathrm{S} / \mathrm{D}$ & 1,1 & 0,8 & $\mathrm{~S} / \mathrm{D}$ & $\mathrm{S} / \mathrm{D}$ & 1,4 & 1,1 & 0,8 & 0,7 & 2,8 & 1,4 & $11,5 \quad 9,1$ \\
\hline GOT & 271 & 154 & 80 & 18 & 271 & $x$ & 670 & 67 & 146 & 56 & 118 & 31 & 84 & 42 & 127 & 34 & 265 & 42 & $186 \quad 101$ \\
\hline GTP & 300 & S/D & 41 & 41 & 288 & $x$ & 1751 & 79 & 182 & 62 & 116 & 10 & 88 & 36 & 348 & 59 & 179 & 34 & 199 \\
\hline TP & $35 \%$ & $29 \%$ & $68 \%$ & $\mathrm{~S} / \mathrm{D}$ & $38 \%$ & $x$ & $52 \%$ & $81 \%$ & $44 \%$ & $64 \%$ & $64 \%$ & $72 \%$ & $35 \%$ & $52 \%$ & $70 \%$ & $68 \%$ & $60 \%$ & $72 \%$ & $54 \% \quad 68 \%$ \\
\hline
\end{tabular}

Tabla 6. Evolución clínica de los pacientes durante el seguimiento

\begin{tabular}{|c|c|c|c|c|c|c|c|c|c|c|}
\hline & Caso $1^{(1)}$ & Caso 2 & Caso $3^{(2)}$ & Caso $4^{(3)}$ & Caso 5 & Caso 6 & Caso 7 & Caso $8^{(4)}$ & Caso 9 & Caso 10 \\
\hline MEJORÍA & & $\checkmark$ & & & $\checkmark$ & $\checkmark$ & & & $\checkmark$ & $\checkmark$ \\
\hline RECAÍDA & & & & $\checkmark$ & & & $\checkmark$ & & & \\
\hline $\begin{array}{l}\text { ABANDONO DE } \\
\text { TRATAMIENTO }\end{array}$ & $\checkmark$ & & $\checkmark$ & & & & & $\checkmark$ & & \\
\hline MUERTE & $\checkmark$ & & $\checkmark$ & & & & & & & \\
\hline
\end{tabular}

\section{DISCUSIÓN}

A pesar de que la HAI sea una enfermedad poco frecuente en pediatría, es una de las causas más frecuentes de hepatopatía crónica en este grupo ${ }^{(18)}$.

Comparando con un trabajo retrospectivo realizado en nuestro centro de estudio en el año 2000, en el que 36 pacientes tenían el diagnóstico de hepatopatía crónica, la HAI representaba el 28\% (10 casos) de los pacientes, $11 \%$ ( 4 pacientes) post hepatitis B y $28 \%$ (10 pacientes) de causa genética o metabólica y en $33 \%$ (12 pacientes) no se pudo definir la etiología ${ }^{(19)}$. La introducción en el Programa Ampliado de Inmunización (PAI) de la vacuna contra los virus Hepatitis A y B pudiera haber influido en la disminución de hepatopatías crónicas causadas por estos virus.

A diferencia de las publicaciones revisadas, en nuestra serie se mostró una predominancia del sexo masculino con una relación 4:1 en el grupo etario de 9 a 15 años ${ }^{(3,20)}$.

Es importante reconocer las alteraciones tanto clínicas como laboratoriales de la enfermedad, como la hepatomegalia, el aumento de bilirrubina y de las transaminasas que se pudieron objetivar en el presente trabajo, y a partir de allí iniciar el estudio de causas de hepatopatías ${ }^{(1,3,12,20)}$.

En este estudio, el 50\% de los pacientes presentaron anticuerpos negativos, y por lo tanto, fueron catalogados como hepatitis autoinmune no clasificada, no habiendo diferencia en la forma de presentación clínica ni en la respuesta al tratamiento, comparado con aquellos que sí presentaron anticuerpos positivos.

Dos pacientes presentaron el antecedente de infección previa por el Virus de la Hepatitis A, con marcadores serológicos Positivos, que fueron los únicos casos en los que pudimos demostrar un probable disparador de la enfermedad. En un solo caso se demostró asociación a otra enfermedad autoinmune, como el LES, lo cual puede presentarse de hecho en asociación con cualquier enfermedad autoinmune, tales como tiroiditis autoinmune, enfermedad inflamatoria intestinal, artritis idiopática juvenil, vitiligo, y la enfermedad celíaca, 
más comúnmente ${ }^{(21-24)}$. Los antecedentes familiares de enfermedad autoinmune se presentan en aproximadamente el $40 \%$ de los afectados con la HAI, sin embargo, en nuestra casuística no encontramos antecedentes ${ }^{(25)}$.

La biopsia hepática se considera de gran valor para establecer el diagnóstico y para determinar la gravedad de la enfermedad, así como la necesidad de tratamiento ${ }^{(13)}$, sin embargo no se implementó en forma sistemática en nuestros pacientes debido a dificultades referentes a la situación clínica de los pacientes y a la disponibilidad de equipos en el hospital.

La totalidad de los pacientes inició tratamiento con prednisona y azatioprina como se menciona en otros estudios ${ }^{(1,3,12,18)}$, independientemente de la clasificación asignada y de los niveles de aminotransferasas. Se observó un alto porcentaje de respuesta al tratamiento médico $(70 \%)$ en nuestra serie, con mejoría progresiva en los parámetros bioquímicos de función hepática en las primeras 6 semanas de tratamiento.

En cuanto a la adherencia al tratamiento, se observó abandono y/o seguimiento irregular en el $40 \%$ de los casos. Es importante indicar el alto porcentaje de recaídas durante el tratamiento observado en un estudio mexicano ${ }^{(26)}$ que fue del $45 \%$, lo cual obligó a realizar ajuste de dosis de esteroides y agregar

\section{REFERENCIAS}

1. Czaja AJ, Manns MP. Advances in the diagnosis, pathogenesis, and management of autoimmune hepatitis. Gastroenterology. 2010;139(1):58-72.

2. Mieli-Vergani G, Heller S, Jara P, Vergani D, Chang MH, Fujisawa T, González-Peralta RP, Kelly D, Mohan N, Shah U, Murray KF. Autoimmune hepatitis. J Pediatr Gastroenterol Nutr. 2009;49:158-64.

3. Luxon BA. Diagnosis and treatment of autoimmune hepatitis. Gastroenterol Clin N Am. 2008;37:461-78.

4. Bjornsson E, Talwalkar J, Treeprasertsuk S, Kamath PS, Takahashi N, Sanderson S, Neuhauser M, Lindor K. Druginduced autoimmune hepatitis: clinical characteristics and prognosis. Hepatology. 2010;51(6):2040-48. terapéutícas más específicas. Otro aspecto a tener en cuenta es el acompañamiento psicológico en el marco interdisciplinario, para el cumplimiento del tratamiento ya que la HAI constituye una enfermedad crónica.

Tres pacientes abandonaron el tratamiento, 2 de los cuales fallecieron por falla hepática y uno abandonó también el seguimiento. En cuanto al resto de los pacientes se pudo descender la prednisona hasta dosis mínima e incluso suspenderla, continuando con azatioprina, en todos los casos sin biopsia hepática de control, solo en base a parámetros clínicos y de laboratorio. Es importante recordar que el $55 \%$ de los pacientes en remisión bioquímica completa todavía tendrá actividad histológica y sus posibilidades de recaída superan el $75 \%{ }^{(25)}$. En caso de falla terapéutica, se mencionan la posibilidad del cambio de prednisona a budesonide, y de azatioprina a micofenolato mofetil, ciclosporina, con diferentes resultados ${ }^{(27-29)}$.

Las dificultades tanto para el diagnóstico como para el tratamiento adecuado, radican en la falta de medios económicos de nuestra población y la ausencia de estudios específicos y medicación en salud pública. Es perentoria la creación y ejecución de presupuestos estatales para solventar el gasto que generan las enfermedades huérfanas en la población de escasos recursos.

5. Efe C, Purnak T, Ozaslan E, Wahlin S. Drug-induced autoimmune hepatitis caused by anti-tumor necrosis factor alpha agents. Hepatology. 2010;52(6):2246-47.

6. Ramakrishna J, Johnson AR, Banner BF. Long-term minocycline use for acne in healthy adolescents can cause severe autoimmune hepatitis. J Clin Gastroenterol. 2009;43(8):787-90.

7. Alvarez F, Berg PA, Bianchi FB, Bianchi L, Burroughs AK, Cancado EL et al. International Autoimmune Hepatitis Group Report: review of criteria for diagnosis of autoimmune hepatitis. J Hepatol. 1999;31:929-38.

8. Hennes EM, Zeniya M, Czaja AJ, Parés A, Dalekos GN, Krawitt EL, Bittencourt PL, Porta G, Boberg KM, Hofer H, 
Bianchi FB, Shibata M, Schramm C, Eisenmann de Torres B, Galle PR, McFarlane I, Dienes HP, Lohse AW. Simplified criteria for the diagnosis of autoimmune hepatitis. Hepatology. 2008;48:169-76.

9. Mileti E, Rosenthal P, Peters MG. Validation and modification of simplified diagnostic criteria for autoimmune hepatitis in children. Clin Gastroenterol Hepatol. 2012;10:417-21.

10. Mieli-Vergani G, Vergani D. Autoimmune pediatric liver disease. World J Gastroenterol. 2008;14(21):3360-67.

11. Bogdanos DP, Invernizzi P, Mackay IR, Vergani D. Autoimmune liver serology: current diagnostic and clinical challenges. World J Gastroenterol. 2008;14(21):3374-87.

12.Teufel A, Galle PR, Kanzler S. Update on autoimmune hepatitis. World J Gastroenterol. 2009;15(9):1035-41.

13. Dezsofi A, Baumann U, Dhawan A, Durmaz O, Fischler B, Hadzic N, Hierro L, Lacaille F, McLin VA, Nobili V, Socha P, Vajro P, Knisely AS. Liver Biopsy in Children: position paper of the ESPGHAN Hepatology Committee. JPGN. 2015;60(3):408-20.

14. Di Giorgio A, Bravi M, Bonanomi E, Alessio G, Sonzogni A, Zen Y, Colledan M, D'Antiga L. Fulminant hepatic failure of autoimmune aetiology in children. JPGN. 2015;60:159-64.

15. Chai PF, Lee WS, Brown RM, McPartland JL, Foster K, McKiernan PJ, Kelly DA. Childhood autoimmune liver disease: indications and outcome of liver transplantation. JPGN. 2010;50:295302.

16. Word Health Organization. The WHO child growth standards [Internet]. Ginebra: Word Health Organization; 2015 [actualizado 2015 Ene; citado 2015 Ene 10]. Disponible en: http://www.who.int/childgrowth/

17. Colli A, Franquelli M, Andreoletti M, Marino B, Zuccoli M, Conte D. Severe liver fibrosis or cirrhosis: accuracy of US for detection-analysis of 300 cases. Radiology. 2003;227:89-94.

18. Cuarteroloa M, Cioccab M, Álvarez F. Hepatitis autoinmune en niños: perspectivas actuales. Arch Argent Pediatr. 2014;112(2):169-75.
19. Garcete L, Corrales B. Evaluación, etiología y clínica de la hepatopatía crónica en niños. Revista de la Sociedad de Gastroenterología del Perú. 2001;21(4):39.

20. Low J, Tan M, García A, Aw M, Quak S. Chilhood autoinmune hepatitis in a pediatric unit of a tertiary care hospital. Singapore Med J. 2014;55(12);648-51.

21. Efe C, Purnak T, Ozaslan E. Concurrent autoimmune thyroid diseases in patients with autoimmune hepatitis. J Clin Gastroenterol. 2010;44(9):660-61.

22. Mirzaagha F, Azali SH, Islami F, Zamani F, Khalilipour E, Khatibian M, Malekzadeh R. Coeliac disease in autoimmune liver disease: a cross-sectional study and a systematic review. Dig Liver Dis. 2010;42(9):620-23.

23. Teufel A, Weinmann A, Kahaly GJ, Centner C, Piendl A, Worns M, Lohse AW, Galle PR, Kanzler S. Concurrent autoimmune diseases in patients with autoimmune hepatitis. J Clin Gastroenterol. 2010;44(3):208-13.

24. Beisel C, Weiler-Normann C, Teufel A, Lohse A. Association of autoimmune hepatitis and systemic lupus erythematodes: a case series and review of the literature. World Journal of Gastroenterology. 2014;21;20(35):12662-67.

25. Mayo MJ. Management of autoimmune hepatitis. Curr Opin Gastroenterol. 2011;27(3):224-30.

26. Nares-Cisneros J, Jaramillo-Rodríguez Y. Hepatitis autoinmune en niños: evolución de 20 casos del Norte de México. Revista de Gastroenterología de México. 2014;79(4):238-43.

27. Snider KR, Potter TG. Budesonide for the treatment of autoimmune hepatitis. Ann Pharmacother. 2011;45(9):1144-50.

28. Hennes EM, Oo YH, Schramm C, Denzer U, Buggisch P, Wiegard C, Kanzler S, Schuchmann M, Boecher W, Galle PR, Adams DH, Lohse AW. Mycophenolate mofetil as second line therapy in autoimmune hepatitis? Am J Gastroenterol. 2008;103(12):3063-70.

29. Zaja Franulovic O, Rajacic N, Lesar T, Tesija Kuna A, Moric V. Cyclosporine induced biochemical remission in childhood autoimmune hepatitis. Coll Antropol. 2012;36(3):973-79. 